\section{Complex Quaternion Representation of Proper Lorentz Transformations}

\section{K. O. Thielheim}

Institut für Reine und Angewandte Kernphysik Kiel (Z. Naturforsch. 24 a, 1858 [1969] ; received 3 October 1969)

The representation of the proper Lorentz group by nonsingular complex quaternions is, of course, a quite obvious one, since it is well known ${ }^{1}$ that spatial rotations may be represented by real quaternions, while velocity transformations may be identified with rotations by imaginary angles, which, therefore, may be represented by quaternions with imaginary vector part and real scalar part. Since any proper Lorentz transformation is the product of one velocity transformation with one spatial rotation, the representation of this group by nonsingular complex quaternions is established. We whish to cast this representation in a form useful for applications.

1. Any $x \in Q_{\mathrm{c}}$ with $x^{*} x=S\left(x^{*} x\right)$ is of the form $x=x\{\mathfrak{r}+i t\}$ with $x \in C, \mathfrak{r} \in E_{3}, t \in R$. Furthermore,

$$
x \rightarrow x^{\prime}=q x \tilde{q},
$$

with $q q^{+} \neq 0$, is a linear mapping of this subspace on itself, which leaves the scalar product $(x, y)=-S\left(x^{*} y\right)$ invariant. Moreover, if $\operatorname{Im}\left(q q^{*}\right)=0$, $\arg \varkappa$ is also left invariant and (1) represents a Lorentz transformation $\mathrm{r}+i t \rightarrow \mathrm{r}^{\prime}+i t^{\prime}$

2. Any $q \in Q_{\mathrm{c}}$ is of the form ${ }^{2}$

$$
q=\lambda q_{1} q_{2},
$$

with $\lambda \in C, \quad q_{j}=\mathfrak{q}_{j} \sqrt{1-\gamma_{j}} / \sqrt{2}+\sqrt{1+\gamma_{j}} / \sqrt{2}, \quad \mathfrak{a}_{j} \in E_{3}$, $\left(\mathfrak{q}_{j}, \mathfrak{q}_{j}\right)=1, \gamma_{j} \in R,-1<\gamma_{2} \leqq+1,+1 \leqq \gamma_{1}<+\infty$, which may be interpreted as follows.

$q=\mathfrak{q} \sqrt{1-\gamma} / \sqrt{ } 2+\sqrt{1+\gamma / \sqrt{2}}$ with $\mathfrak{q} \in E_{3}, \quad(\mathfrak{q}, \mathfrak{q})=1$, $-1<\gamma \leqq+1$ is a real normalized quaternion, there-

Reprint requests to Doz. Dr. K. O. Thielheim, Institut für Reine und Angewandte Kernphysik der Universität Kiel, D-2300 Kiel, Olshausenstraße 40-60.

1 W.BlaschKe, Kinematik und Quaternionen, VEB Deutscher Verlag der Wissenschaften, Berlin 1960; P. Rastall, Rev. Mod. Phys. 36, 820-832 [1964]. Gives references to earlier work, e. g. F. KLEIN, Phys. Z. 12, 17 [1911]. - Multiplication of quaternions is usually introduced by means of a multiplication table $u_{j} u_{k}=\varepsilon_{j k l} u_{l}-\delta_{j k}, u_{0} u_{j}=u_{j} u_{0}=u_{j}$, $u_{0}^{2}=u_{0}$, for the basis quaternions with $j, k, l \in 1,2,3$. Matrix realizations are easily constructed with the help of infinitesimal generators $I_{j}$ of any matrix representation of fore, $q=q^{-1}$. As is well known, $x \rightarrow x^{\prime}=q x q^{-1}$ is a spatial rotation with rotation vector $=\mathfrak{q} \operatorname{arc} \cos \gamma$.

$q=\mathfrak{q} \sqrt{1-\gamma} / \sqrt{2}+\sqrt{1+\gamma / \sqrt{2}}$ with $\mathfrak{q} \in E_{3}, \quad(\mathfrak{q}, \mathfrak{q})=1$, $+1 \leqq \gamma<+\infty$ is a complex normalized quaternion with $\operatorname{Re} V(q)=\operatorname{Im} S(q)=0$, therefore, $\tilde{q}=q$. As is also well known, $x \rightarrow x^{\prime}=q x q$ is a velocity transformation with velocity vector $\mathfrak{v}=q \sqrt{\gamma^{2}-1} / \gamma$, namely

$$
\begin{gathered}
\mathfrak{r} \rightarrow \mathfrak{r}^{\prime}=[\mathfrak{v},[\mathfrak{r}, \mathfrak{v}]] / \mathfrak{v}^{2}+\mathfrak{v}\left\{t+(\mathfrak{v}, \mathfrak{r}) / \mathfrak{v}^{2}\right\} / \sqrt{1-\mathfrak{v}^{2}}, \\
t \rightarrow t^{\prime}=\{t+(\mathfrak{v}, \mathfrak{r})\} / \sqrt{1-\mathfrak{v}^{2}} .
\end{gathered}
$$

Thus by (1) and (2) with $\lambda=1$, we have reestablished the representation of the proper Lorentz group by normalized complex quaternions, where any transformation is given by the product of one velocity transformation with one rotation.

3. Eq. (2) may be applied to any product $q=q_{3} q_{4}$, with $-1<\gamma_{3}, \gamma_{4}<+\infty$, in which case $\lambda=1$. The result is trivial if $-1<\gamma_{3} \leqq+1,+1 \leqq \gamma_{4}<+\infty$ or vice versa, and almost trivial if $-1<\gamma_{3}, \gamma_{4} \leqq+1$, since in the latter case the product of two rotations is one rotation times the identity velocity transformation. If $+1 \leqq \gamma_{3}, \gamma_{4}<+\infty$, the product of two velocity transformations is given ${ }^{2}$ by the product of one rotation by the rotation vector

$$
\begin{aligned}
\boldsymbol{\delta}_{2}= & \frac{\left[\mathfrak{v}_{3}, \mathfrak{v}_{4}\right]}{\left|\left[\mathfrak{v}_{3}, \mathfrak{v}_{4}\right]\right|} \\
& \cdot \operatorname{arc} \cos \left\{1-\frac{\left(1+\gamma_{3}\right)\left(1+\gamma_{4}\right)\left[1+\gamma_{3} \gamma_{4}\left(1+\left(\mathfrak{v}_{3}, \mathfrak{v}_{4}\right)\right)\right]}{\left[\mathfrak{v}_{3}, \mathfrak{v}_{4}\right]^{2} \gamma_{3}{ }^{2} \gamma_{4}{ }^{2}}\right\}
\end{aligned}
$$

with one velocity transformation by the velocity

$$
\mathfrak{v}_{1}=\frac{\mathfrak{v}_{3}+\mathfrak{v}_{4} / \gamma_{3}+\mathfrak{v}_{3}\left(\mathfrak{v}_{3}, \mathfrak{v}_{4}\right)\left(\gamma_{3}-1\right) / \gamma_{3} \mathfrak{v}_{3}{ }^{2}}{1+\left(\mathfrak{v}_{3}, \mathfrak{v}_{4}\right)}
$$

which, of course, is just the theorem of velocity addition.

$\mathrm{SO}(3, \mathrm{R}), u_{j}=-i I_{j}$, e. g. irreducible representations with weight $l$, the most familiar ones are those with weight $l=\frac{1}{2}$ $\left(I_{j}=\sigma_{j}, \quad\right.$ Pauli-matrices) and $l=1, \quad\left(I_{j}\right)_{k l}=-i \varepsilon j k l$. The following notation is used. $C$ : field of complex numbers, $R$ : field of real numbers, $Q_{\mathrm{c}}$ : field of complex quaternions, $E_{3}$ : three dimensional euklidean space, $V(q)$ and $S(q)$ are the vector part and the scalar part, respectively, of a quaternion $q=V(q)+S(q)$. $q^{*}$ is obtained from $q$ by complex conjugation of coefficients.

$q^{+}=-V(q)+S(q), \quad q^{-1}=q^{+} / q q^{+}, \quad \tilde{q}=\left(q^{*}\right)^{-1}=\left(q^{-1}\right)^{*}$.

2 This is easily verified by performing the multiplications and comparing real and imaginary coefficients.

\title{
BERICHTIGUNG
}

$\mathrm{Zu}$ W. Scobel, Wirkungsquerschnitt der Reaktion ${ }^{9} \mathrm{Be}(\mathrm{n}, \mathrm{d}){ }^{8} \mathrm{Li}$ zwischen $16,3 \mathrm{MeV}$ und 18,7 MeV, Z. Naturforsch. 24 a, 289 [1969].

Der Ordinatenmaßstab in Abb. 2 ist um einen Faktor 5/3 falsch; der höchste eingetragene Wert muß entsprechend 10 (statt 6) mb heißen.

Nachdruck - auch auszugsweise - nur mit schriftlicher Genehmigung des Verlages gestattet

Verantwortlich für den Inhalt: A. KLEMM

Satz und Druck: Konrad Triltsch, Würzburg 